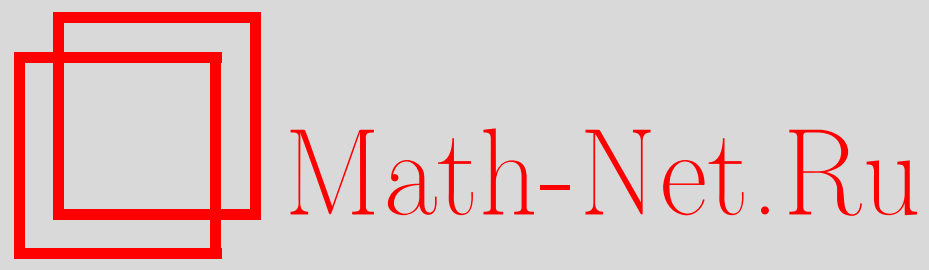

Ю. В. Чеканов, Критические точки квазифункций и производящие семейства лежандровых многообразий, Функи. анализ и его прил., 1996, том 30, выпуск 2, 56-69

DOI: https://doi.org/10.4213/faa521

Использование Общероссийского математического портала MathNet.Ru подразумевает, что вы прочитали и согласны с пользовательским соглашением

http://www . mathnet.ru/rus/agreement

Параметры загрузки:

IP : 3.93 .64 .190

26 апреля 2023 г., 07:08:41

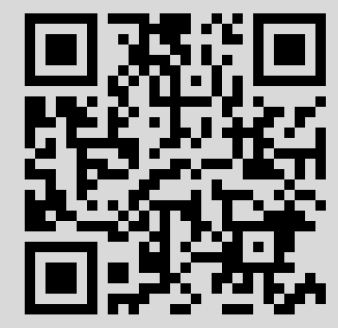


Функииональньй анализ и еәо приложения

1996, т. 30, вып. 2, с. 56-69

УДК 517.9

\title{
Критические точки квазифункций и производящие семейства лежандровых многообразий
}

\author{
(c) 1996. Ю. В. ЧЕКАнов ${ }^{1}$
}

Классические теоремы Люстерника-Шнирельмана и Морса дают оценку снизу числа критических точек гладкой функции на замкнутом многообразии $M$ через (ко)гомологии этого многообразия. Эти результаты можно интерпретировать как утверждения о числе точек пересечения точного (т.е. такого, ограничение на которое формы Лиувилля точно) лагранжева сечения кокасательного расслоения $T^{*} M$ с нулевым сечением: вместо функции надо рассмотреть ее дифференциал. Нетривиальный факт заключается в том, что оценка останется верной, если заменить точное лагранжево сечение произвольным точным вложенным лагранжевым многообразием, гомотопным нулевому сечению в классе точных вложенных лагранжевых многообразий. Это утверждение, высказанное в качестве гипотезы Арнольдом [1], было доказано Лоденбаком и Сикоравом [7] (а чуть раньше - для случая $M=T^{n}$ - Шапроном [6]).

В настоящей работе доказывается, в частности, дальнейшее обобщение теорем Люстерника-Шнирельмана и Морса, в котором вместо точных лагранжевых подмногообразий кокасательного расслоения участвуют лежандровы подмногообразия расслоения 1-струй. При этом ключевую роль играет теорема, гласящая, что в пространстве вложенных лежандровых многообразий множество многообразий, заданных квадратичным на бесконечности производящим семейством, открыто и замкнуто в $C^{\infty}$-топологии (аналогичное утверждение для лагранжева случая было доказано Сикоравом [8]).

Основные результаты работы были анонсированы автором в 1986-1987 гг. (см. $[3,10])$. Доказательства рассказывались, в частности, на семинаре В. И. Арнольда в МГУ. С тех пор некоторые конструкции (такие, как формула композиции и трюк с вложением) вошли в симплектический фольклор. Полные доказательства публикуются, насколько известно автору, впервые.

\section{§1. Критические точки квазифункций}

Напомним вначале некоторые стандартные определения и конструкции контактной топологии (см. также $[2,4])$. Пусть $M$ - гладкое многообразие. Расслоение 1-струй $J^{1} M=T^{*} M \times \mathbb{R} \rightarrow M$ есть прямая сумма кокасательного расслоения $T^{*} M \rightarrow M$ и тривиального расслоения 0 -струй $J^{0} M=(M \times \mathbb{R}) \rightarrow M$. Многообразие $J^{1} M$ снабжено контактной формой $\alpha=d u-p d q$, где $u-$ стандартная координата на $\mathbb{R}$, а $p d q-$ форма Лиувилля на $T^{*} M$. Подмногообразие размерности $n$ контактного многообразия размерности $2 n+1$ (скажем, $J^{1} M$,

\footnotetext{
${ }^{1}$ Работа выполнена при частичной поддержке ISF, грант MSD300, и INTAS, грант 4373.
} 
где $\operatorname{dim} M=n)$ называется лежандровым, если ограничение на него контактной формы равно нулю.

По гладкой функции $f: M \rightarrow \mathbb{R}$ можно построить сечение $j^{1} f$ расслоения 1-струй (называемое 1-графиком функции $f$ ) как прямую сумму сечения $d f$ расслоения $T^{*} M \rightarrow M$ (дифференциала функции $f$ ) и сечения $j^{0} f$ расслоения $J^{0} M$ (графика функции $f$ ). Легко проверить, что 1-графики функций на $M-$ это в точности лежандровы подмногообразия многообразия $J^{1} M$, являющиеся (гладкими) сечениями. Обозначим через $M_{0}$ нулевое сечение расслоения $T^{*} M$ и через $\pi$ естественную проекцию $J^{1} M\left(=T^{*} M \times \mathbb{R}\right) \rightarrow T^{*} M$. Точку $x$ лежандрова многообразия $\Lambda$ мы называем критической, если $\pi(x) \in M_{0}$; если к тому же пересечение $\Lambda$ с $\pi^{-1}\left(M_{0}\right)$ трансверсально в $x$, мы называем критическую точку $x$ морсовской (невырожденной). Это определение обобщает определение критической точки функции: если $\Lambda=j^{1} f$, то критические точки многообразия $\Lambda$ переходят при проекции на $M$ в критические точки функции $f$ (и морсовские точки переходят в морсовские).

Квазифункиией на замкнутом многообразии $M$ называется вложенное лежандрово подмногообразие пространства $J^{1} M$, (гладко) гомотопное нулевому сечению $j^{1} 0 \subset J^{1} M$ в классе лежандровых вложений.

Пусть $A$ - коммутативное кольцо. Напомним, что когомологической длиной $\operatorname{cl}(M, A)$ многообразия $M$ называется наибольшее число $k$, для которого найдутся такие когомологические классы $\eta_{1}, \ldots, \eta_{k} \in H^{>0}(M, A)$, что их произведение $\eta_{1} \cdots \eta_{n}$ отлично от нуля. Следующая теорема обобщает стандартные теоремы Люстерника-Шнирельмана и Морса (и сводится к ним в случае $\left.\Lambda=j^{1} f\right)$.

ТЕорема 1.1. Пусть $\Lambda$ - квазифункиия на замкнутом многообразии $M$. Тогда число ее критических точек

(а) не меньше чем $\operatorname{cl}(M, A)+1$;

(б) при условии, что все критические точки невырожденнь, не меньше чем

$$
\sum_{i=0}^{\operatorname{dim} M}\left(b_{i}(M)+2 q_{i}(M)\right),
$$

аде $b_{i}(M)=\operatorname{dim} H_{i}(M, \mathbb{R})$ и $q_{i}(M)$ - минимальное число образующих аруппь Tors $H_{i}(M, \mathbb{Z})$.

Рассмотрим теперь расслоение 1-струй функций со значениями в $S^{1}=\mathbb{R} / \mathbb{Z}$ : $J^{1}\left(M, S^{1}\right)=T^{*} M \times S^{1} \rightarrow M$. Пространство $J^{1}\left(M, S^{1}\right)=\left(J^{1} M\right) / \mathbb{Z}$ наследует контактную структуру от $J^{1} M$. Аналогично случаю $J^{1} M$ мы называем точку $x$ лежандрова многообразия $\Lambda$ критической, если $\hat{\pi}(x) \in M_{0}\left(\right.$ где $\hat{\pi}: J^{1}\left(M, S^{1}\right) \rightarrow$ $T^{*} M$ - естественная проекция), и морсовской, если пересечение $\Lambda$ с $\hat{\pi}^{-1}\left(M_{0}\right)$ трансверсально в $x$.

Проекции $J^{1}\left(M, S^{1}\right) \rightarrow T^{*} M, J^{1}\left(M, S^{1}\right) \rightarrow M$ определяют по всякому лежандрову сечению $\Lambda_{0}$ расслоения $J^{1}\left(M, S^{1}\right) \rightarrow M 1$-форму $\beta_{\Lambda_{0}} \in H^{1}(M, \mathbb{R})$ и функцию $f_{\Lambda_{0}}: M \rightarrow S^{1}$, причем $\beta_{\Lambda_{0}}=d f_{\Lambda_{0}}$; следовательно, форма $\beta_{\Lambda_{0}}$ целочисленна. Ее нули соответствуют критическим точкам сечения $\Lambda_{0}$. Обозначим через $\xi$ когомологический класс $\left[\beta_{\Lambda_{0}}\right] \in H^{1}(M, \mathbb{Z}) /$ Tors. Обобщением теоремы 1.1(б) (и одновременно неравенств Новикова, оценивающих число нулей 
замкнутой 1-формы в предположении их невырожденности [9]) является следующая

ТЕОРема 1.2. Пусть $\Lambda$ - лежандрово подмногообразие многообразия $J^{1}\left(M, S^{1}\right)$, гладко гомотопное в классе вложенных лежандровых многообразий некоторому сечению $\Lambda_{0}$. Тогда число критических точек многообразия $\Lambda$ при условии их невырожденности не меньше чем

$$
\sum_{i=0}^{\operatorname{dim} M}\left(b_{i}(M, \xi)+2 q_{i}(M, \xi)\right),
$$

где $b_{i}(M, \xi)$ - ранг, а $q_{i}(M, \xi)$ - число кручения группь гомологий Новикова $H_{i}(M, \xi)$.

\section{§2. Производящие семейства для квазифункций}

Если лежандрово многообразие $\Lambda \subset J^{1} M$ есть 1-график функции $f: M \rightarrow \mathbb{R}$, то $f$ называется производящей функцией многообразия $\Lambda$. Обобщением понятия производящей функщии является понятие производящего семейства. Теорема 1.1 есть следствие того факта, что всякая квазифункция может быть задана производящим семейством специального вида (квадратичным на бесконечности).

Напомним определение производящего семейства (см. [4]). Пусть $M$ и $W$ - гладкие многообразия, а $F: M \times W \rightarrow \mathbb{R}$ - гладкая функция. Обозначим канонические координаты на $T^{*} M$ через $(p, q)$, а на $T^{*} W-$ через $(v, w)$; тогда

$$
j^{1} F=\left\{(p, v, q, w, u) \mid p=F_{q}(q, w), v=F_{w}(q, w), u=F(q, w)\right\} .
$$

Пусть $W_{0}$ - нулевое сечение расслоения $T^{*} W$ и $E=J^{1} M \times W_{0} \subset J^{1}(M \times W)$. Предположим, что пересечение $j^{1} F$ с $E$ трансверсально, что равносильно следующему условию на $F$ :

$$
\text { если } F_{w}(q, w)=0 \text {, то } \operatorname{rk}\left(F_{q w}(q, w), F_{w w}(q, w)\right)=\operatorname{dim} W .
$$

Тогда $X=j^{1} F \cap E-$ гладкое многообразие той же размерности, что и $M$. Так как $X \subset j^{1} F$, то форма $d u-p d q-v d w$ обращается в нуль на $X$. Поскольку $v=0$ на $E$, форма $d u-p d q$ также обращается в нуль на $X$. Следовательно, проекция многообразия $X$ на $J^{1} M-$ (иммерсированное) лежандрово многообразие

$$
\Lambda=\left\{(p, q, u) \mid \exists w \in W: F_{w}(q, w)=0, p=F_{q}(q, w), u=F(q, w)\right\} .
$$

При выполнении условия (2.2) функция $F$ называется производящим семейством лежандрова многообразия $\Lambda$ (и лагранжева многообразия $\pi(\Lambda) \subset T^{*} M$ ).

Одно и то же лежандрово многообразие может задаваться различными производящими семействами. Важным примером является следующий: если $F: M \times W \rightarrow \mathbb{R}$ - производящее семейство, то $F \circ D$, где $D$ - расслоенный над $M$ диффеоморфизм $M \times W$, есть производящее семейство, задающее то же лежандрово многообразие, что и $F$. 
Из (2.3) следует, что критические точки лежандрова многообразия $\Lambda$, заданного производяшим семейством $F: M \times W \rightarrow \mathbb{R}$, 一 это в точности образы критических точек функции $F$ при отображении

$$
M \times W \rightarrow J^{1} M, \quad(q, w) \mapsto(0, q, F(q, w)) .
$$

Если многообразие $\Lambda$ вложенное, то это отображение задает взаимно однозначное соответствие между критическими точками функции $F$ и критическими точками многообразия $\Lambda$.

ПРЕДЛОЖЕНИЕ 2.1. Критическая точка $x$ лежандрова многообразия $\Lambda$ является морсовской тогда и только тогда, когда соответствующал ей критическая точка $(q, w)$ прочзводящего семейства $F$ морсовская.

ДокАЗАтЕльСтво. Рассмотрим симплектическое линейное пространство $(Y, \omega)=\left(T_{y} T^{*}(M \times W)\right)$, где $y=(0,0, q, w), \omega$ - естественная симплектическая форма кокасательного расслоения. Его подпространства $L_{1}=T_{y}(d F)$, $L_{2}=T_{y}\left(M_{0}, W_{0}\right)$ лагранжевы, а подпространство $V=T_{y}\left(T^{*} M \times W_{0}\right)$ коизотропно (т.е. $\operatorname{dim} \operatorname{ker}\left(\left.\omega\right|_{V}\right)=\operatorname{codim} V$ ). Операция симплектической редукции относительно $V$ (заключающаяся во взятии пересечения с $V$ и последующей проекции вдоль $\left.\operatorname{ker}\left(\left.\omega\right|_{V}\right)=T_{y} W_{0}\right)$ переводит $Y$ в симплектическое пространство $Y^{\prime}=T_{(0, q)} T^{*} M$, а лагранжевы подпространства $L_{1}, L_{2} \subset Y-$ в лагранжевы подпространства $L_{1}^{\prime}=\pi_{*}\left(T_{x} \Lambda\right), L_{2}^{\prime}=T_{q} M_{0} \subset Y^{\prime}$. Невырожденность $(q, w)$ равносильна трансверсальности подпространств $L_{1}$ и $L_{2}$, а невырожденность $x$ - трансверсальности $L_{1}^{\prime}$ и $L_{2}^{\prime}$. Предложение 2.1 немедленно вытекает из следующего элементарного факта линейной алгебры:

Лемма 2.2. Пусть $V$ - коизотропное подпространство симплектического линейного пространства $Y$. Лагранжевы подпространства $L_{1}, L_{2} \subset Y$ трансверсальны тогда и только тогда, когда трансверсальны лагранжевь подпространства, полученнье из них симплектической редукиией относительно $V$.

Пусть многообразие $M$ замкнуто. Функция $F: M \times \mathbb{R}^{l}$ называется квадратичной на бесконечности, если она имеет вид

$$
F(q, w)=\varphi(q, w)+Q(w),
$$

где $\varphi$ - финитная функция, а $Q$ - невырожденная квадратичная форма. Лежандрово многообразие мы называем правильным, если оно задается квадратичным на бесконечности производящим семейством.

ТЕОРема 2.3. Всякая квазифункиия есть правильное лежандрово многообразие.

Теорема 1.1 есть следствие теоремы 2.3 и следующей классической теоремы Конли-Цендера:

Теорема 2.4 [5]. Пусть $M$ - замкнутое многообразие, $F: M \times \mathbb{R}^{l}-$ квадратичная на бесконечности функция и $A$ - коммутативное кольчо. Тогда число критических точек $F$

(а) не меньше чем $\mathrm{cl}(M, A)+1$;

(б) не меньше чем

$$
\sum_{i=0}^{\operatorname{dim} M}\left(b_{i}(M)+2 q_{i}(M)\right),
$$


при условии, что все критические точки морсовские.

Поскольку нулевое сечение $J^{1} M$ - правильное лежандрово многообразие (задаваемое нулевой производящей функцией), теорема 2.3 является частным случаем следующей теоремы:

ТЕОРема 2.5. Пусть $\left\{\Lambda_{t}\right\}_{t \in[0,1]}$ - гладкое семейство вложенньх лежандровых подмногообразий пространства $J^{1} M$. Тогда если $\Lambda_{0}$ правильное, то и $\Lambda_{1}$ правильное.

Иначе говоря, в пространстве лежандровых многообразий множество правильных открыто и замкнуто в $C^{\infty}$-топологии. Эта теорема доказывается в $\S \S 3-5$. В $\S 6$ с использованием полученных в $\S 3-5$ результатов доказывается теорема 1.2 .

\section{§3. Трюк с вложением: сведение к случаю $M=\mathbb{R}^{k}$}

Вместо теоремы 2.5 нам будет удобно доказывать следующее эквивалентное ей утверждение:

ТЕОрема 3.1. Пусть $M$ - замкнутое многообразие $u\left\{G^{t}\right\}_{t \in[0,1]}$ - гладкое семейство контактоморфизмов пространства $J^{1} M, G^{0}=\mathrm{id}$. Если лежандрово многообразие $\Lambda_{0} \subset J^{1} M$ является правильньл, то и лежандрово многообразие $\Lambda_{1}=G^{1}\left(\Lambda_{0}\right)$ правильное.

Эта теорема есть лежандров аналог теоремы Сикорава [8]. Чтобы показать, что теорема 2.5 следует из теоремы 3.1, достаточно построить такое семейство $\left\{v_{t}\right\}_{t \in[0,1]}$ финитных контактных векторных полей на $J^{1} M$, что $\Lambda_{t}=G^{t}\left(\Lambda_{0}\right)$, где $G^{t}$ - отображение за время от 0 до $t$ фазового потока, заданного семейством $\left\{v_{t}\right\}$. При помощи разбиения единищы на $[0,1]$, подчиненного покрытию достаточно короткими интервалами, задача сводится к случаю, когда все многообразия $\Lambda_{t} C^{1}$-близки к $\Lambda_{0}$. Контактно отождествим окрестность $U$ многообразия $\Lambda_{0}$ с окрестностью нулевого сечения в $J^{1} \Lambda_{0}$; тогда $\Lambda_{t}$ отождествится с $j^{1} f_{t}$, где $\left\{f_{t}\right\}$ - некоторое семейство функций на $\Lambda_{0}$. Напомним, что для любого многообразия $N$ существует взаимно однозначное соответствие между функциями на $J^{1} N$ и контактными векторными полями на $\left(J^{1} N, d u-p d q\right)$ : полю

$$
v=\left(K_{q}+p K_{u}\right) \partial / \partial p-K_{p} \partial / \partial q+\left(K-p K_{p}\right) \partial / \partial u
$$

соответствует функция $K$, называемая контактным гамильтонианом поля $v$ $[2,4]$. Искомое поле $v_{t}$ может быть задано при помощи контактного гамильтониана $k^{t}$, который обращается в нуль вне $U$, а в окрестности $\Lambda_{t}$ совпадает с $\dot{f}_{t} \circ \rho$, где $\rho: J^{1} \Lambda_{0} \rightarrow \Lambda_{0}$ - естественная проекция. Эквивалентность теоремы 2.5 и теоремы 3.1 установлена.

Мы покажем, что теорема 3.1 есть следствие аналогичного ей утверждения, в котором вместо замкнутого многообразия $M$ участвует пространство $\mathbb{R}^{k}$. Определение квадратичной на бесконечности функции в случае $V=\mathbb{R}^{k}$ будет несколько иным. Функция $F: \mathbb{R}^{k} \times \mathbb{R}^{l}$ называется квадратичной на бесконечности, если $F(q, w)=\varphi(q, w)+Q(w)$, где $\varphi$ - функция с ограниченными первыми производными, а $Q$ - невырожденная квадратичная форма. 
Теорема 3.2. Пусть $\left\{g^{t}\right\}_{t \in[0,1]}$ - гладкое семейство контактоморфизмов пространства $J^{1} \mathbb{R}^{k}$, непрерывное в $C^{\infty}$-топологии, $g^{0}=\mathrm{id}$. Если лежандрово многообразие $\Theta_{0} \in J^{1} \mathbb{R}^{k}$ задается квадратичным на бесконечности производящим семейством, то и лежандрово многообразие $\Theta_{1}=g^{1}\left(\Theta_{0}\right)$ задается квадратичным на бесконечности производячим семейством.

Эта теорема доказывается в $\S 5$ с использованием результатов, полученных в $§ 4$. Оставшаяся часть настоящего параграфа посвящена выводу теоремы 3.1 из теоремы 3.2 .

Зафиксируем вложение $M \hookrightarrow \mathbb{R}^{k}$ и положим $J_{M}^{1} \mathbb{R}^{k}=\rho^{-1}(M)$, где $\rho-$ отображение расслоения $J^{1} \mathbb{R}^{k} \rightarrow \mathbb{R}^{k}$. Пусть $I: J_{M}^{1} \mathbb{R}^{k} \rightarrow J^{1} \mathbb{R}^{k}$ и $P: J_{M}^{1} \mathbb{R}^{k} \rightarrow$ $J^{1} M$ - естественные отображения вложения и проекции. Будем называть контактоморфизмы $G: J^{1} M \rightarrow J^{1} M$ и $g: J^{1} \mathbb{R}^{k} \rightarrow J^{1} \mathbb{R}^{k}$ согласованными, если $g\left(J_{M}^{1} \mathbb{R}^{k}\right)=J_{M}^{1} \mathbb{R}^{k}$ и следующая диаграмма (в которой средняя стрелка - сужение $g$ на $\left.J_{M}^{1} \mathbb{R}^{k}\right)$ коммутативна:

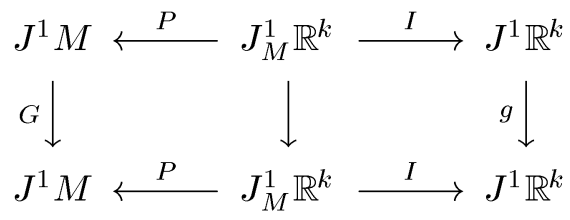

ПРЕДЛОЖЕНИЕ 3.3. Пусть $\left\{G^{t}\right\}_{t \in[0,1]}$ - гладкое семейство контактоморфизмов пространства $J^{1} M$, тождественных вне компакта, $G^{0}=\mathrm{id}$. Тогда найдется такое гладкое семейство $\left\{g^{t}\right\}_{t \in[0,1]}$ контактоморфизмов пространства $J^{1} \mathbb{R}^{k}$, непрерьвное в $C^{\infty}$-топологии, что $g^{0}=\mathrm{id}$ и при любом $t \in[0,1]$ контактоморфизмь $G^{t} u g^{t}$ согласованьл.

ДокАЗАТЕльСТво. Пусть $\left\{K^{t}\right\}_{t \in[0,1]}$ - семейство контактных гамильтонианов на $J^{1} M$, соответствуюших векторным полям $V^{t}=\dot{G}^{t}$. Продолжим семейство (определенных на $J_{M}^{1} \mathbb{R}^{k}$ ) функций $\left\{K^{t} \circ P\right\}$ до семейства $\left\{k^{t}\right\}$ функций на $J^{1} \mathbb{R}^{k}$, которые $C^{m}$-ограничены для любого $m$. Тогда при любом $t \in[0,1]$ векторное поле $v^{t}$, заданное контактным гамильтонианом $k^{t}$, касается многообразия $J_{M}^{1} \mathbb{R}^{k}$ и проектируется в поле $V^{t}$ под действием $P$ (это легко проверить, записав $v^{t}$ в локальных координатах, в которых $M$ выделяется прямым сомножителем). Порожденное семейством $v^{t}$ семейство контактоморфизмов пространства $J^{1} \mathbb{R}^{k}$ будет искомым.

Пусть $\Sigma$ - лежандрово подмногообразие пространства $J^{1} \mathbb{R}^{k}$. Если пересечение $\Sigma$ с $J_{M}^{1} \mathbb{R}^{k}$ трансверсально, то $\Sigma_{M}=P\left(\Sigma \cap J_{M}^{1} \mathbb{R}^{k}\right)$ - (иммерсированное) лежандрово подмногообразие многообразия $J^{1} M$. В этом случае мы называем $\Sigma_{M}$ лежандровым ограничением $\Sigma$ на $J^{1} M$.

ПрЕДЛОЖЕНИЕ 3.4. Пусть $F: \mathbb{R}^{k} \times W \rightarrow \mathbb{R}$ - производящее семейство лежандрова многообразия $\Sigma \subset J^{1} \mathbb{R}^{k}$. Сужение $F^{M}$ функиии $F$ на $M \times$ $W$ является производяиим семейством тогда и только тогда, когда $\Sigma$ трансверсально пересекает $J_{M}^{1} \mathbb{R}^{k} ;$ в этом случае лежандрово ограничение многообразия $\Sigma$ на $J^{1} M$ задается производящим семейством $F^{M}$.

ДокАЗАТЕЛЬСтво. Первое утверждение вытекает из леммы 2.2. Второе легко проверяется непосредственным вычислением в локальных координатах, 
в которых $M$ выделяется прямым сомножителем. Пусть

Приступим теперь непосредственно к выводу теоремы 3.1 из теоремы 3.2 .

$$
f^{0}: M \times \mathbb{R}^{l} \rightarrow \mathbb{R}, \quad(q, w) \mapsto \varphi_{0}(q, w)+Q_{0}(w)
$$

- квадратичное на бесконечности производящее семейство для $\Lambda_{0}$. Продолжим $\varphi_{0}$ до гладкой функции $\Phi_{0}$ на $\mathbb{R}^{k} \times \mathbb{R}^{l}$ с ограниченными первыми производными так, чтобы функция

$$
F^{0}: \mathbb{R}^{k} \times \mathbb{R}^{l} \rightarrow \mathbb{R}, \quad(q, w) \mapsto \Phi_{0}(q, w)+Q_{0}(w)
$$

была производящим семейством некоторого лежандрова многообразия $\Theta_{0}$ (это несложно сделать, используя сильную теорему трансверсальности). Согласно предложению $3.4, \Lambda_{0}$ есть лежандрово ограничение $\Theta_{0}$ на $J^{1} M$. Построим семейство $\left\{g^{t}\right\}$ контактоморфизмов пространства $J^{1} \mathbb{R}^{k}$, удовлетворяющее условиям предложения 3.3. По теореме $3.2 \Theta_{1}$ задается квадратичным на бесконечности производяшим семейством $F^{1}: \mathbb{R}^{k} \times \mathbb{R}^{m} \rightarrow \mathbb{R}$. Сужение $f^{1}$ производящего семейства $F^{1}$ на $M \times \mathbb{R}^{m}$ является, согласно предложению 3.4 , производящим семейством для $\Lambda_{1}$. При этом

$$
f^{1}(q, w)=\varphi(q, w)+Q(w)
$$

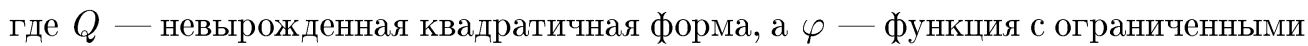
первыми производными (в любой метрике, являющейся прямой суммой метрики на $M$ и евклидовой метрики на $\left.\mathbb{R}^{m}\right)$, но, вообще говоря, не финитная. Нам осталось показать, что производящее семейство $f^{1}$ может быть приведено к сумме финитной функции и квадратичной формы $Q(w)$ при помощи диффеоморфизма многообразия $M \times \mathbb{R}^{m}$, расслоенного над $M$. Воспользуемся гомотопическим методом. Положим

$$
h_{t}(q, w)=(1-t) \varphi(q, w)+Q(w) ;
$$

тогда найдется семейство векторных полей $\left\{v_{t}\right\}$ на $M \times \mathbb{R}^{m}$, которые всюду касаются слоев расслоения и удовлетворяют гомологическому уравнению $L_{v_{t}} h_{t}=\dot{h}_{t}$ вне некоторого компакта. Отображение за время 1 фазового потока, заданного семейством $\left\{v_{t}\right\}$, является искомым диффеоморфизмом.

\section{§4. Композиция симплектических соответствий и производящие семейства}

В этом параграфе мы изучаем связь между композицией симплектических соответствий (например, композицией симплектоморфизмов) и производящими семействами. Основной результат — теорема 4.1 - идейно восходит к работе Шапрона [6].

Пусть $\left(X_{1}, \omega_{1}\right)$ и $\left(X_{2}, \omega_{2}\right)$ - симплектические многообразия. Лагранжевы подмногообразия симплектического многообразия $\left(X_{1} \times X_{2},\left(-\omega_{1}\right) \oplus \omega_{2}\right)$ будем называть симплектическими соответствиями. Так же, как соответствие обобщает понятие отображения, симплектическое соответствие обобщает понятие симплектического отображения: график отображения $g: X_{1} \rightarrow X_{2}$ есть симплектическое соответствие тогда и только тогда, когда $g$ - симплектоморфизм. 
Пусть $\left(X_{i}, \omega_{i}\right), i \in\{0,1,2\}$, - симплектические многообразия, а $U_{01} \subset$ $X_{0} \times X_{1}, U_{12} \subset X_{1} \times X_{2}$ - симплектические соответствия. Композиция $U_{02}=$ $U_{12} \circ U_{01} \subset X_{0} \times X_{2}$ симплектических соответствий $U_{01}, U_{12}$ определяется как образ при проекции

$$
\Pi: X_{0} \times X_{1} \times X_{1} \times X_{2} \rightarrow X_{0} \times X_{2}
$$

пересечения прямого произведения $U_{01} \times U_{12}$ с многообразием

$$
\Delta=X_{0} \times \Delta_{1} \times X_{2} \subset X_{0} \times X_{1} \times X_{1} \times X_{2},
$$

где $\Delta_{1} \subset X_{1} \times X_{1}$ - диагональ.

Будем называть пару $U_{01}, U_{12}$ хорошей, если пересечение $U_{01} \times U_{12}$ с $\Delta$ трансверсально. В этом случае $U_{02}$ - (иммерсированное) лагранжево многообразие (так как $U_{02}$ есть симплектическая редукция лагранжева многообразия $U_{01} \times U_{12}$ относительно коизотропного многообразия $\left.\Delta\right)$. Несложно проверить, что если $U_{01}$ или $U_{12}$ - график симплектоморфизма, то пара $U_{01}, U_{12}$ хорошая. Если $U_{01}$ и $U_{12}$ - графики симплектоморфизмов, то $U_{12} \circ U_{01}$ - график композиции этих симплектоморфизмов. Если $X_{0}=\{\mathrm{pt}\}$ и $U_{12}$ - график симплектоморфизма $g: X_{1} \rightarrow X_{2}$, то $U_{01}$ можно представлять себе как лагранжево подмногообразие в $X_{1}$; тогда $U_{12} \circ U_{01}$ есть лагранжево подмногообразие $g\left(U_{01}\right)$ многообразия $X_{2}$ (с такой ситуацией мы будем иметь дело в $\left.\S 5\right)$.

Пусть теперь $\left(X_{i}, \omega_{i}\right)=\left(T^{*} \mathbb{R}^{k_{i}}, d p \wedge d q\right), i=1,2$. Зафиксируем разложения

$$
\begin{gathered}
T^{*} \mathbb{R}^{k_{i}}=\left\{\left(p_{i}, q_{i}\right) \mid p_{i} \in\left(\mathbb{R}^{k_{i}}\right)^{*}, q_{i} \in \mathbb{R}^{k_{i}}\right\}, \\
V=\left(\mathbb{R}^{k_{1}}\right)^{*} \times \mathbb{R}^{k_{2}}, \quad V^{*}=\mathbb{R}^{k_{1}} \times\left(\mathbb{R}^{k_{2}}\right)^{*} .
\end{gathered}
$$

Рассмотрим кокасательное расслоение $T^{*} V=V^{*} \times V$, снабженное канонической симплектической формой $\omega=d P \wedge d Q$, где $P=\left(q_{1}, p_{2}\right) \in V^{*}, Q=\left(p_{1}, q_{2}\right) \in V$. Отображение

$$
\left(X_{1} \times X_{2},\left(-\omega_{1}\right) \oplus \omega_{2}\right) \rightarrow\left(T^{*} V, \omega\right), \quad\left(p_{1}, q_{1}, p_{2}, q_{2}\right) \mapsto\left(q_{1}, p_{2}, p_{1}, q_{2}\right)
$$

- симплектоморфизм, поскольку $d P \wedge d Q=d p_{2} \wedge d q_{2}-d p_{1} \wedge d q_{1}$.

Итак, в рассматриваемом случае симплектические соответствия отождествляются с лагранжевыми подмногообразиями кокасательного расслоения. Значит, они могут быть заданы производящими семействами. Замечательный факт заключается в том, что композиция симплектических соответствий, заданных производящими семействами, также задается производящим семейством, которое явно выражается через производящие семейства исходных симплектических соответствий.

Имея в виду дальнейшие приложения, мы расширим понятие производящего семейства, позволив ему (при выполнении условия трансверсальности (2.2)), быть определенным на открытом подмножестве $\Omega \subset\left(\mathbb{R}^{k_{1}}\right)^{*} \times \mathbb{R}^{k_{2}} \times W$. Производяшее семейство $S: \Omega \rightarrow \mathbb{R}$ задает лагранжево многообразие (симплектическое соответствие) $L \subset T^{*} \mathbb{R}^{k_{1}} \times T^{*} \mathbb{R}^{k_{2}}$ :

$$
\begin{array}{r}
L=\left\{\left(p_{1}, q_{1}, p_{2}, q_{2}\right) \mid \exists w:\left(p_{1}, q_{2}, w\right) \in \Omega, S_{w}\left(p_{1}, q_{2}, w\right)=0,\right. \\
\left.p_{2}=S_{q_{2}}\left(p_{1}, q_{2}, w\right), q_{1}=S_{p_{1}}\left(p_{1}, q_{2}, w\right)\right\} .
\end{array}
$$


Рассмотрим симплектические пространства

$$
\left(X_{i}, \omega_{i}\right)=\left(\left\{\left(p_{i}, q_{i}\right)\right\}=\left(\mathbb{R}^{k_{i}}\right)^{*} \times \mathbb{R}^{k_{i}}, d p_{i} \wedge d q_{i}\right),
$$

где $i \in\{0,1,2\}$. Пусть $L_{01} \subset X_{0} \times X_{1}, L_{12} \subset X_{1} \times X_{2}$ - хорошая пара симплектических соответствий. Предположим, что $L_{01}$ задается производящим семейством $S^{01}: \Omega_{01} \rightarrow \mathbb{R}, \Omega_{01} \subset\left(\mathbb{R}^{k_{0}}\right)^{*} \times \mathbb{R}^{k_{1}} \times W_{01}$, а $L_{12}$ - производящим семейством $S^{12}: \Omega_{12} \rightarrow \mathbb{R}, \Omega_{12} \subset\left(\mathbb{R}^{k_{1}}\right)^{*} \times \mathbb{R}^{k_{2}} \times W_{12}$. Обозначим через $\sigma$ отображение перестановки

$$
\left(p_{0}, q_{1}, w_{01}, p_{1}, q_{2}, w_{12}\right) \mapsto\left(p_{0}, q_{2}, w_{01}, w_{12}, p_{1}, q_{1}\right) .
$$

Теорема 4.1. Композииия симплектических соответствий $L_{02}=L_{12} \circ L_{01}$ задается производяшим семейством $S^{02}: \Omega_{02} \rightarrow \mathbb{R}$, где $\Omega^{02}=\sigma\left(\Omega^{01} \times \Omega^{12}\right)$,

$$
S^{02}\left(p_{0}, q_{2}, w_{01}, w_{12}, p_{1}, q_{1}\right)=S^{01}\left(p_{0}, q_{1}, w_{01}\right)+S^{12}\left(p_{1}, q_{2}, w_{12}\right)-p_{1} q_{1} .
$$

ДокАЗАТЕЛЬСтво. Тот факт, что $L_{02}$ есть образ $\left(L_{01} \times L_{12}\right) \cap \Delta$ при проекции на $X_{0} \times X_{2}$, несложно проверить, выписав выражения (4.2) для $L_{01}, L_{12}$, $L_{02}$. Тот факт, что $S^{02}$ есть производящее семейство, получается применением аналога леммы 2.2 , в котором многообразия $L_{2}, L_{2}^{\prime}$ коизотропные, а не лагранжевы.

\section{$\S 5$. Симплектизация и производящие семейства}

Мы доказываем теорему 3.2 при помощи метода симплектизации, сводящего задачу о лежандровых подмногообразиях контактного многообразия к задаче о лагранжевых подмногообразиях симплектического многообразия. Ключевым моментом доказательства является применение теоремы 4.1 к производящим семействам симплектизованных объектов.

Пусть $(C, \alpha)$ - контактное многообразие. Рассмотрим прямое произведение $C \times \mathbb{R}_{\times}$, где $\mathbb{R}_{\times}$- группа ненулевых действительных чисел; пусть $\sigma: C \times$ $\mathbb{R}_{\times} \rightarrow C$ и $\lambda: C \times \mathbb{R}_{\times} \rightarrow \mathbb{R}_{\times}$- естественные проекции. Симплектическое многообразие

$$
\left(S C, \omega_{\alpha}\right)=\left(C \times \mathbb{R}_{\times},-d\left(\lambda \sigma^{*} \alpha\right)\right)
$$

мы называем симплектизацией контактного многообразия $(C, \alpha)$. Наше определение отличается от стандартного (см. $[2,4])$ знаком симплектической формы. Слоями расслоения $\sigma$ являются орбиты действия группы $\mathbb{R}_{\times}$на $S C=C \times \mathbb{R}_{\times}$ (тривиального на первом сомножителе и регулярного на втором). Симплектическая форма $\omega_{\alpha}$ однородна степени 1 относительно этого действия.

Пусть $\Lambda \subset C$ - лежандрово многообразие. Его симплектизацией называется многообразие $\sigma^{-1}(\Lambda) \subset S C$. Если $g: C \rightarrow C$ - контактоморфизм, то $\alpha=m g^{*} \alpha$ для некоторой функщии $m: C \rightarrow \mathbb{R}$, называемой мультипликатором контактоморфизма $g$. Симплектизацией $S g$ контактоморфизма $g$ называется диффеоморфизм пространства симплектизации $S C$, задаваемый формулой

$$
S g(x, \lambda)=(g(x), m(x) \lambda) .
$$

ПреДложЕнИЕ $5.1[2,4]$. (а) Отображение $\Lambda \mapsto S \Lambda$ задает взаимно однозначное соответствие между лежандровыми многообразиями в $C$ и $\mathbb{R}_{\times}$-инвариантными лагранжевыми многообразиями в $S C$. 
(б) Отображение $g \mapsto S g$ задает взаимно однозначное соответствие между контактоморфизмами многообразия $C$ и симплектоморфизмами многообразия $S C$, коммутируюшими с действием $\mathbb{R}_{\times}$.

Основное свойство процедуры симплектизации заключается в том, что по контактному (лежандрову) объекту строится симплектический (лагранжев) объект, удовлетворяющий некоторому условию $\mathbb{R}_{\times}$-эквивариантности. Симплектизация пространства 1-струй оказывается кокасательным расслоением:

ПрЕДлОЖенИЕ 5.2. Симплектизация контактного многообразия $\left(J^{1} N\right.$, $d u-p d q)$ канонически симплектоморфна пространству $T^{*}\left(N \times \mathbb{R}_{\times}\right)$, снабженному стандартной симплектической формой $\omega$.

ДокАЗАТЕЛЬСТво. Диффеоморфизм

$$
T^{*}\left(N \times \mathbb{R}_{\times}\right) \rightarrow J^{1} N \times \mathbb{R}_{\times}, \quad(p, u, q, \lambda) \mapsto(\lambda p, q, u, \lambda)
$$

переводит форму $\omega=d p \wedge d q+d u \wedge d \lambda$ в форму $-d(\lambda(d u-p d q))$.

Симплектизация $S \Theta$ лежандрова подмногообразия $\Theta$ пространства 1-струй есть лагранжево подмногообразие кокасательного расслоения. Как $\Theta$, так и $S \Theta$, могут, вообще говоря, задаваться производящими семействами. Следующее важное утверждение устанавливает связь между производящими семействами лежандрова многообразия и его симплектизации.

ПреДЛОЖЕНИЕ 5.3. ФУнкиия $F: N \times W \rightarrow \mathbb{R}$ является производящим семейством лежандрова многообразия $\Theta \subset J^{1} N$ тогда и только тогда, когда функция

$$
S F:\left(N \times \mathbb{R}_{\times}\right) \times W \rightarrow \mathbb{R}, \quad(q, \lambda, w) \mapsto \lambda F(q, w)
$$

является производящим семейством лагранжева многообразия $S \Theta \subset T^{*}(N \times$ $\left.\mathbb{R}_{\times}\right)$.

ДокАЗАТЕЛЬСтво. Если одна из функций $F, S F$ удовлетворяет условию трансверсальности (2.2), то и другая тоже, так как ранги матриц

$$
\left(S F_{q w}, S F_{\lambda w}, S F_{w w}\right)=\lambda\left(F_{q w}, F_{w}, F_{w w}\right) \quad \text { и } \quad\left(F_{q w}, F_{w w}\right)
$$

совпадают в точках, где $F_{w}=0$. Производящее семейство $F$ задает лежандрово многообразие

$$
\Theta=\left\{(p, q, u) \mid \exists w \in W: F_{w}=0, p=F_{q}, u=F\right\},
$$

а производящее семейство $S F$ - лагранжево многообразие

$$
T=\left\{(p, q) \mid \exists w \in W: F_{w}=0, p=\lambda F_{q}\right\} .
$$

Поскольку проекция $\sigma: T^{*}\left(N \times \mathbb{R}_{\times}\right) \rightarrow J^{1} N$ в координатах имеет вид

$$
\sigma(p, u, q, u)=\left(\lambda^{-1} p, q, u\right),
$$

мы получаем $T=\sigma^{-1}(\Theta)$. 
Отметим, что действие группы $\mathbb{R}_{\times}$на $T^{*}\left(N \times \mathbb{R}_{\times}\right)$(обозначим его $A$ ) имеет вид

$$
A_{\mu}(p, u, q, \lambda)=(\mu p, u, q, \mu \lambda) .
$$

Рассмотрим подробнее интересуюший нас случай $N=\mathbb{R}^{k}$. Вложение $T^{*}\left(\mathbb{R}^{k} \times \mathbb{R}_{\times}\right) \hookrightarrow T^{*}\left(\mathbb{R}^{k} \times \mathbb{R}\right)$ симплектично; поэтому график $\Gamma$ контактоморфизма $g: J^{1} \mathbb{R}^{k} \rightarrow J^{1} \mathbb{R}^{k}$ есть лагранжево подмногообразие симплектического многообразия $(X, \omega)=\left(X_{1} \times X_{2},\left(-\omega_{1}\right) \oplus \omega_{2}\right)$, где

$$
X_{i}=\left(T^{*}\left(\mathbb{R}^{k} \times \mathbb{R}\right)\right)_{i}=\left(\mathbb{R}^{k *}\right)_{i} \times\left(\mathbb{R}^{*}\right)_{i} \times\left(\mathbb{R}^{k}\right)_{i} \times \mathbb{R}_{i}=\left\{\left(p_{i}, u_{i}, q_{i}, \lambda_{i}\right)\right\},
$$

$\omega_{i}=d p_{i} \wedge d q_{i}+d u_{i} \wedge d \lambda_{i}$. Отображение (4.1) симплектически отождествляет $X$ c $T^{*} V=V^{*} \times V$, где

$$
V=\left(\mathbb{R}^{k^{*}}\right)_{1} \times\left(\mathbb{R}^{*}\right)_{1} \times\left(\mathbb{R}^{k}\right)_{2} \times \mathbb{R}_{2}, \quad V^{*}=\left(\mathbb{R}^{k}\right)_{1} \times \mathbb{R}_{1} \times\left(\mathbb{R}^{k^{*}}\right)_{2} \times\left(\mathbb{R}^{*}\right)_{2} .
$$

Определим действие группы $\mathbb{R}_{\times}$на $V, V^{*}, T^{*} V=X$ :

$$
\begin{aligned}
A_{\mu}^{V}\left(p_{1}, u_{1}, q_{2}, \lambda_{2}\right) & =\left(\mu p_{1}, u_{1}, q_{2}, \mu \lambda_{2}\right), \\
A_{\mu}^{V^{*}}\left(q_{1}, \lambda_{1}, p_{2}, u_{2}\right) & =\left(q_{1}, \mu \lambda_{1}, \mu p_{2}, u_{2}\right), \\
A_{\mu}^{T^{*} V}\left(v^{*}, v\right) & =\left(A_{\mu}^{V^{*}}\left(v^{*}\right), A_{\mu}^{V}(v)\right) .
\end{aligned}
$$

Форма $\omega$ однородна степени 1 относительно действия $A^{T^{*} V}$.

ПредЛОЖенИЕ 5.4. График симплектизации контактоморфизма пространства $J^{1} \mathbb{R}^{k}$ инвариантен относительно действия $A^{T^{*} V}$ группь $\mathbb{R}_{\times} \cdot$

ДокаЗАТЕЛЬСТво. Множество

$$
X_{1}^{\times} \times X_{2}^{\times}=\left\{\lambda_{1} \lambda_{2} \neq 0\right\}=\left(T^{*}\left(\mathbb{R}^{k} \times \mathbb{R}\right)\right)_{1} \times\left(T^{*}\left(\mathbb{R}^{k} \times \mathbb{R}\right)\right)_{2} \subset X_{1} \times X_{2}
$$

инвариантно относительно этого действия. Из формул (5.1) и (5.2) видно, что $A^{T^{*} V}\left(x_{1}, x_{2}\right)=\left(A_{\mu}\left(x_{1}\right), A_{\mu}\left(x_{2}\right)\right)$ при $\left(x_{1}, x_{2}\right) \in X_{1}^{\times} \times X_{2}^{\times}$. Поэтому предложение 5.4 следует из предложения 5.2 .

График $\gamma$ симплектоморфизма пространства $T^{*} \mathbb{R}^{k}, C^{1}$-близкого к тождественному, задается производящей функцией вида $S\left(p_{1}, q_{2}\right): \gamma=\left\{q_{1}=S_{p_{1}}\right.$, $\left.p_{2}=S_{q_{2}}\right\}$. Хотя симплектизация нетождественного контактоморфизма не близка к диагонали, аналогичный результат справедлив и в нашем случае. Соответствующая производящая функция будет определена на множестве $V^{\prime}=\left(\mathbb{R}^{k}\right)_{1} \times$ $\left(\mathbb{R}^{*}\right)_{1} \times\left(\mathbb{R}^{k}\right)_{2} \times\left(\mathbb{R}_{\times}\right)_{2} \subset V$, инвариантном относительно действия $A^{V}$.

ПредЛОЖЕНИЕ 5.5. График $\Gamma \subset T^{*} V$ симплектизачии $C^{2}$-близкого $\kappa$ тождественному контактоморфизма $g: J^{1} \mathbb{R}^{k} \rightarrow J^{1} \mathbb{R}^{k}$ есть дифференциал некоторой функиии $D: V^{\prime} \rightarrow \mathbb{R}$, удовлетворяющей условию $\mathbb{R}_{\times}$-однородности $D \circ A_{\mu}^{V}=\mu D$.

ДокАЗАТЕЛЬСТво. Покажем, что проекция $\pi_{V}: T^{*} V \rightarrow V$ диффеоморфно отображает $\Gamma$ на $V^{\prime}$. Положим

$$
V_{1}=\left(\mathbb{R}^{k^{*}}\right)_{1} \times\left(\mathbb{R}^{*}\right)_{1} \times\left(\mathbb{R}^{k}\right)_{2} \times\{1\} \subset V, \quad Z=\pi_{v}^{-1}\left(V_{1}\right) .
$$

В силу $C^{2}$-близости $g$ к id пересечение $\Gamma$ с $Z C^{1}$-близко к пересечению диагонали с $Z$; поэтому проекция $\Gamma \cap Z$ на $V_{1}$ - диффеоморфизм. Отсюда следует ввиду предложения 5.4, что $\Gamma$ есть сечение расслоения $T^{*} V^{\prime} \rightarrow V^{\prime}$ и, 
значит, задается некоторой производящей функцией $D: V^{\prime} \rightarrow \mathbb{R}$. Из условия $\mathbb{R}_{\times}$-инвариантности $\Gamma$ вытекает, что $d\left(D \circ A_{\mu}^{V}-\mu D\right)=0$ при любом $\mu \in \mathbb{R}_{\times}$. Прибавив к $D$ подходящую локально постоянную функцию, получим $D \circ A_{\mu}^{V}=$ $\mu D$ при $\mu= \pm 1$, а значит, и при всех $\mu \in \mathbb{R}_{\times}$.

Приступим к доказательству теоремы 3.2. Ее достаточно доказать в предположении $C^{2}$-малости контактоморфизма $g^{1}$ (общий случай сводится к этому разбиением отрезка $[0,1])$. Пусть лежандрово многообразие $\Theta_{0} \subset\left(J^{1} \mathbb{R}^{k}\right)_{1}$ задано квадратичным на бесконечности производящим семейством

$$
F^{0}:\left(\mathbb{R}^{k}\right)_{1} \times \mathbb{R}^{l} \rightarrow \mathbb{R}, \quad\left(q_{1}, w\right) \mapsto \varphi\left(q_{1}, w\right)+Q(w) .
$$

Согласно предложению 5.3, его симплектизация $S \Theta_{0} \subset\left(T^{*}\left(\mathbb{R}^{k} \times \mathbb{R}\right)\right)_{1}$ задается производящим семейством

$$
S F^{0}:\left(\mathbb{R}^{k}\right)_{1} \times\left(\mathbb{R}_{\times}\right)_{1} \times \mathbb{R}^{l} \rightarrow \mathbb{R}, \quad\left(q_{1}, \lambda_{1}, w\right) \mapsto \lambda_{1}\left(\varphi\left(q_{1}, w\right)+Q(w)\right) .
$$

График $\Gamma$ симплектизации контактоморфизма $g^{1}$ задается, согласно предложению 5.5 , производящей функцией $D: V^{\prime} \rightarrow \mathbb{R}$. Применяя теорему $4.1 \mathrm{k}$ паре симплектических соответствий $S \Theta_{0}, \Gamma$ (в нашем случае $X_{0}=\{\mathrm{pt}\}, X_{1}=$ $\left.\left(T^{*}\left(\mathbb{R}^{k} \times \mathbb{R}\right)\right)_{1}, X_{2}=\left(T^{*}\left(\mathbb{R}^{k} \times \mathbb{R}\right)\right)_{2}, W_{01}=\mathbb{R}^{l}, W_{12}=\{\mathrm{pt}\}\right)$, получаем, что лагранжево многообразие $S \Theta_{1}=S g^{1}\left(S \Theta_{0}\right) \subset\left(T^{*}\left(\mathbb{R}^{k} \times \mathbb{R}_{\times}\right)\right)_{2}$ задается производящим семейством $F^{1}:\left(T^{*}\left(\mathbb{R}^{k} \times \mathbb{R}_{\times}\right)\right)_{2} \times W_{1} \rightarrow \mathbb{R}$, где

$$
\begin{gathered}
W_{1}=\left\{\left(w, p_{1}, u_{1}, q_{1}, \lambda_{1}\right)\right\}=\mathbb{R}^{l} \times\left(T^{*}\left(\mathbb{R}^{k} \times \mathbb{R}_{\times}\right)\right)_{1}, \\
F^{1}\left(q_{2}, \lambda_{2}, w_{1}\right)=\lambda_{1} F^{0}\left(q_{1}, w\right)+D\left(p_{1}, u_{1}, q_{2}, \lambda_{2}\right)-p_{1} q_{1}-u_{1} \lambda_{1} .
\end{gathered}
$$

Мы хотели бы теперь, воспользовавшись предложением 5.5, найти производящее семейство для лежандрова многообразия $\Theta_{1}$. Поскольку $F^{1}$ имеет неподходящий вид, мы заменим его другим производящим семейством, задающим то же лагранжево многообразие $S \Theta_{1}$. Сначала превратим $W_{1}$ в линейное пространство. Пусть $b>a>0$ и $\tau: \mathbb{R} \rightarrow \mathbb{R}$ — такая неубывающая гладкая функция, что $\tau(x)=x$ при $x \in[a, b], \tau(x)=a / 2$ при $x \leqslant a / 2, \tau(x)=2 b$ при $x \geqslant 2 b$.

Лемма 5.6. Найдутся такие $a, b$, что функиия $F^{2}:\left(T^{*}\left(\mathbb{R}^{k} \times \mathbb{R}_{\times}\right)\right)_{2} \times$ $W_{2} \rightarrow \mathbb{R}$, где $W_{2}=\mathbb{R}^{l} \times\left(T^{*}\left(\mathbb{R}^{k} \times \mathbb{R}\right)\right)_{1} \supset W_{1}$,

$$
F^{2}\left(q_{2}, \lambda_{2}, w_{1}\right)=\lambda_{2} \tau\left(\lambda_{1} / \lambda_{2}\right) F^{0}\left(q_{1}, w\right)+D\left(p_{1}, u_{1}, q_{2}, \lambda_{2}\right)-p_{1} q_{1}-u_{1} \lambda_{1},
$$

является производящим семейством для $S \Theta_{1}$.

ЛЕмма 5.7. Существуют такие числа $b>a>0$, что для любого $x \in V^{\prime}$

$$
a<\lambda_{2}(x)^{-1} D_{u_{1}}(x)<b .
$$

ДокАЗАтЕльство. Число $\lambda_{2}(x)^{-1} D_{u_{1}}(x)$ есть значение мультипликатора контактоморфизма $g^{1}, C^{2}$-близкого к тождественному, в некоторой точке пространства $J^{1} \mathbb{R}^{k}$.

ДОКАЗАТЕЛЬСТВО ЛЕмМЫ 5.6. ВЫберем $a$ и $b$, удовлетворяющие условию леммы 5.7. Положим

$$
B=T^{*}\left(\left(\mathbb{R}^{k} \times \mathbb{R}_{\times}\right)\right)_{2} \times W_{0},
$$


где $W_{0}$ - нулевое сечение $T^{*} W_{2}$. Если $y \in d F_{j} \cap B$, то $F_{u_{1}}^{j}(x)=0$, где $x$ есть образ $y$ при проекции на $V^{\prime}$; поэтому $\lambda_{1}(x)=D_{u_{1}}(x)$. Из леммы 5.7 вытекает теперь, что $d F^{1}$ совпадает с $d F^{2}$ в окрестности многообразия $B$, откуда и следует утверждение леммы.

Рассмотрим диффеоморфизм $s$ многообразия $\left(\left(\mathbb{R}^{k} \times \mathbb{R}_{\times}\right)\right)_{2} \times W_{2}$, расслоенный над $\left(\left(\mathbb{R}^{k} \times \mathbb{R}_{\times}\right)\right)_{2}$ :

$$
\left(q_{2}, \lambda_{2}, w, p_{1}, u_{1}, q_{1}, \lambda_{1}\right) \stackrel{s}{\longmapsto}\left(q_{2}, \lambda_{2}, \tau\left(\lambda_{1}\right)^{-1 / 2} w, \lambda_{2} p_{1}, u_{1}, q_{1}+q_{2}, \lambda_{1} \lambda_{2}\right) .
$$

Производящее семейство $F^{\prime}=F^{2} \circ s$ тоже задает лагранжево многообразие $S \Theta_{1}$. При этом

$$
\begin{aligned}
& F^{\prime}\left(q_{2}, \lambda_{2}, w, p_{1}, u_{1}, q_{1}, \lambda_{1}\right) \\
& \quad=\lambda_{2}\left(\varphi\left(\left(q_{1}+q_{2}\right), \tau\left(\lambda_{1}\right)^{-1 / 2} w\right)+\psi\left(p_{1}, u_{1}, q_{2}\right)+Q(w)-p_{1} q_{1}-u_{1} \lambda_{1}\right),
\end{aligned}
$$

где $\psi\left(p_{1}, u_{1}, q_{2}\right)=D\left(p_{1}, u_{1}, q_{2}, 1\right)$. В соответствии с предложением 5.3 теорема 3.2 вытекает из следующей леммы:

ЛЕмма 5.8. Первые производные функиии $\psi$ ограничены.

ДокАЗАТЕльство. Как отмечалось в доказательстве предложения 5.5, пересечение $d D=\Gamma$ с $Z=\left\{\lambda_{2}=1\right\} C^{1}$-близко к пересечению $d\left(p_{1} q_{2}-u_{1} \lambda_{2}\right)$ с $Z$; поэтому первые производные функции $\psi$ малы.

\section{§6. Доказательство теоремы 1.2}

Мы предполагаем, что класс $\xi$ ненулевой (в противном случае теорема 1.2 легко следует из теоремы 1.1(б)). Пусть $\rho: \bar{M} \rightarrow M-$ регулярное накрытие со слоем $\mathbb{Z}$, для которого $\rho^{*} \xi=0$. Оно определяет контактное накрытие $\rho^{J}: J^{1} \check{M} \rightarrow J^{1} M$. Найдется такая функция $f_{0}$, что $\rho^{J}\left(j^{1} f_{0}\right)=\Lambda_{0}$. Эта функция удовлетворяет условию $\mathbb{Z}$-эквивариантности $f_{0}\left(\theta_{1}(q)\right)=f_{0}(q)+n$, где $\theta_{1}$ - действие образующей группы $\mathbb{Z}$ на $\check{M}$, а число $n$ таково, что $\xi$ есть образующая группы $n H^{1}(M) /$ Tors .

Функцию $F: \check{M} \times W \rightarrow \mathbb{R}$ мы называем $\mathbb{Z}$-эквивариантной, если

$$
F\left(\theta_{1}(q), w\right)=F(q, w)+n,
$$

и квадратичной на бесконечности, если она имеет вид

$$
F(q, w)=f_{0}(q)+\varphi(q, w)+Q(w),
$$

где $\varphi$ - финитная функция, а $Q$ - невырожденная квадратичная форма на $W=\mathbb{R}^{l}$. Лежандрово подмногообразие пространства $J^{1} \bar{M}$ мы называем периодическим, если оно инвариантно относительно действия $\mathbb{Z}$ на $J^{1} \check{M}$, и правильным, если оно задается $\mathbb{Z}$-эквивариантным квадратичным на бесконечности производящим семейством. Теорема 1.2 вытекает из следующих двух утверждений:

Теорема 6.1. Пусть $\left\{\Lambda_{t}\right\}_{t \in[0,1]}$ - гладкое семейство вложенных периодических лежандровых подмногообразий пространства $J^{1} \check{M}$. Тогда если $\Lambda_{0}$ правильное, то и $\Lambda_{1}$ правильное. 
Теорема 6.2. Пусть все критические точки $\mathbb{Z}$-эквивариантной квадратичной на бесконечности функции на $\check{M} \times \mathbb{R}^{l}$ невырожденны; тогда иисло точек проекции критического множества на $M \times \mathbb{R}^{l}$ не меньше

$$
\sum_{i=0}^{\operatorname{dim} M}\left(b_{i}(M, \xi)+2 q_{i}(M, \xi)\right) .
$$

Мы не приводим доказательства теоремы 6.2 ; оно получается скрещиванием доказательств теоремы 2.4(б) (см. [5]) и неравенств Новикова [9].

ДокаЗАтельство теоремы 6.1. Определим действие $\mathbb{Z}$ на $\mathbb{R}^{k}$ так: $\theta_{a}\left(x_{1}, \ldots, x_{k}\right)=\left(x_{1}+a n, \ldots, x_{k}\right)$. Зафиксируем такое вложение $i: \check{M} \hookrightarrow \mathbb{R}^{k}$, коммутирующее с действием $\mathbb{Z}$, что $x_{1} \circ i=f_{0}$. Дальнейшие рассуждения, полностью повторяющие рассуждения $\S 3$, сводят теорему 6.1 к варианту теоремы 3.2 , в котором производящие семейства лежандровых многообразий дополнительно удовлетворяют условию $\mathbb{Z}$-эквивариантности (6.1). После этого остается лишь проверить, что конструкции доказательства теоремы 3.2 при преобразовании старого производящего семейства в новое сохраняют свойство $\mathbb{Z}$-эквивариантности.

ПРИМЕЧАНИЕ ПРИ КОРРЕКТУРЕ. Когда статья уже была подГотовлена к печати, автор получил сведения о том, что теорема 3.2 была доказана М. Шапроном иными методами, см. Chaperon $M$. On generating families. In: Floer memorial volume, Birkhäuser, 1995.

\section{ЛитературА}

1. Arnold V. I. Sur une propriété topologique des applications globalement canonique de la mécanique classique. C. R. Acad. Sci. Paris Sér. I, 261, 3719-3722 (1965).

2. Арнольд В. И. Математические методы классической механики. Наука, М., 1974, дополнение 4.

3. Арнольд В. И. Первые шаги симплектической топологии. УМН, 41, вып. 6, 3-18 (1986).

4. Арнольд В. И., Гивенталь А. Б. Симплектическая геометрия. В кн.: Современные проблемы математики. Динамические системы 4. ВИНИТИ, М., 1986.

5. Conley C., Zehnder E. The Birkhoff-Lewis fixed point theorem and a conjecture of V. I. Arnold. Invent. Math., 73, 33-49 (1983).

6. Chaperon $M$. Une idée du type 'geodesiques brisée' pour les systemes hamiltoniens. C. R. Acad. Sci. Paris Sér. I, 298, 293-296 (1984).

7. Laudenbach F., Sikorav J.-C. Persistance d'intersection avec la section nulle au cours d'une isotopie hamiltonienne dans un fibré cotangent. Invent. Math., 82, 349-358 (1985).

8. Sikorav J.-C. Sur les immersions lagrangiennes dans un fibré cotangent. C. R. Acad. Sci. Paris Sér. I, 32, 119-122 (1986).

9. Новиков С. П. Гамильтонов формализм и многозначный аналог теории Морса. УМН, 37, вып. 5, 3-49 (1982).

10 Чеканов Ю. В. Лежандрова теория Морса. УМН, 42, вып. 4, 139 (1987). 